\title{
The Relationship between the Injury Mechanism and the Incidence of ACL Avulsions in Schatzker Type IV Tibial Plateau Fractures: A 3D Quantitative Analysis Based on Mimics Software
}

\author{
Zihao Liu, MM ${ }^{1}$ Shuai Wang, $\mathrm{MM}^{2}$ Xiaochen Tian, $\mathrm{MM}^{3}$ Aqin Peng, $\mathrm{PhD}^{1}$ (1) \\ ${ }^{1}$ Department of Orthopedic Surgery, Third Hospital of Hebei Medical \\ Address for correspondence Aqin Peng, PhD, Department of \\ University, Shijiazhuang, Hebei, China \\ ${ }^{2}$ Department of Orthopedic Surgery, Hebei Chest Hospital, \\ Shijiazhuang, Hebei, China \\ ${ }^{3}$ Department of Orthopedic Surgery, No. 1 Hospital of Shijiazhuang, \\ Orthopedic Surgery, Third Hospital of Hebei Medical University, No. \\ 139 Ziqiang Road, Shijiazhuang, Hebei, China 050051 \\ (e-mail: PengAqin01022626@163.com).
}

Shijiazhuang, Hebei, China

J Knee Surg 2023;36:644-651.

\begin{abstract}
Keywords

- Schatzker type IV tibial plateau fractures

- ACL avulsions

- quantitative 3D measurements

Introduction Tibial plateau fractures are often accompanied with ligamental and meniscal injuries. Among which, the combined existence of Schatzker type IV fracture with anterior cruciate ligament $(\mathrm{ACL})$ avulsion has been reported rarely. The purpose of this study was to determine the injury mechanism of Schatzker type IV fracture with ACL avulsion based on Mimics software.

Methods Ninety-nine Schatzker type IV tibial plateau fractures were retrospectively analyzed by quantitative three-dimensional measurements. ACL avulsions were diagnosed through the data of computed tomography and magnetic resonance imaging. We simulated the knee posture when an injury occurred and defined different injury patterns. The chi-square test was used for determining the main mechanism which causes Schatzker type IV fractures associated with ACL avulsions.

Results There were more $A C L$ avulsions and more displaced $A C L$ avulsions associated with the knee in flexion in the setting of Schatzker type IV fracture $(p<0.05)$. More ACL avulsions were found in the injury pattern of flexion-valgus than the other injury patterns of the same level $(p<0.05)$. The rotation of the tibial showed no significant difference in producing $A C L$ avulsion fractures.

Conclusion This study found that a flexed knee at the occurrence of a Schatzker type IV tibial plateau fracture is a high-risk factor for causing associated ACL avulsion and producing more displaced avulsions. Flexion-valgus pattern was the main cause of Schatzker type IV fractures associated with $A C L$ avulsions. The findings will help orthopedists understand the injury mechanism and enhance their awareness of such injuries to avoid unfavorable prognosis.
\end{abstract}

Tibial plateau fractures, which are a type of intra-articular injuries, have a wide range of clinical manifestations. ${ }^{1,2}$ To fully describe the injury force mechanism, at least 38 classi- fication systems were proposed by different authors. ${ }^{3}$ Among these systems, the Schatzker classification is widely used in tibial plateau fractures around the world, which is received

January 13, 2021

accepted after revision

November 16, 2021

article published online

January 3, 2022 (c) 2022. Thieme. All rights reserved.

Thieme Medical Publishers, Inc., 333 Seventh Avenue, 18th Floor, New York, NY 10001, USA
DOI https://doi.org/ 10.1055/s-0041-1740929. ISSN 1538-8506. 
divided into six categories. ${ }^{4}$ In the Schatzker classification system, fracture types are gradually classified according to the severity of injury. However, Schatzker later believed that the type IV fracture had the worst prognosis, which is likely due to its high variability and the combination of bony and soft tissue injuries.

Yan et al found that operative Schatzker type IV tibial plateau fractures represented a high incidence of soft-tissue injuries, especially anterior cruciate ligament $(\mathrm{ACL})$ injuries through clinical data analysis. ${ }^{5}$ Yet, ACL avulsion, which is associated with Schatzker type IV tibial plateau fracture would be easily ignored among primary hospitals. ${ }^{6}$ Due to emergent conditions or lack of medical equipment in primary hospitals, orthopedists may ignore the injuries of the ACL by interpreting plain radiographs only. Displaced ACL avulsions often require open or arthroscopic reduction and fixation, while conservative treatments are suggested for nondisplaced ACL avulsions. ${ }^{7}$ The failure of orthopedists to diagnose and treat associated ACL injuries may lead to the long-term poor recovery of knee function accompanying Schatzker type IV tibial plateau fractures..$^{8-10}$

A better understanding of the mechanism of injury can help orthopedists avoid missed diagnoses and offer appropriate treatments. ${ }^{2,11,12}$ Several studies had explored the mechanism of tibial plateau fractures by using computed tomography (CT)-based three-dimensional (3D) models and summarized eight possible injury patterns of Schatzker type IV tibial plateau fractures: flexion-valgus-internal rotation, flexion-valgus-external rotation, flexion-varus-internal rotation, flexion-varus-external rotation, extension-varus, extension-valgus, hyperextension-varus-internal rotation, and hyperextension-valgus-external rotation. ${ }^{11,13}$ The authors have also investigated the correlation between tibial plateau fracture patterns and the injury mechanisms. Wang et $\mathrm{al}^{11}$ found that valgus force was the main cause of the Schatzker type IV tibial plateau fractures and can easily cause soft tissue complications, but the injury mechanism of Schatzker type IV tibial plateau fracture with ACL avulsion has been rarely reported. The aim of this study was to analyze the injury patterns of Schatzker type IV tibial plateau fractures associated with ACL avulsions; this was performed by simulating different knee postures in the setting of fracture occurrence and measuring the rotation angle of tibial.

\section{Materials and Methods}

\section{Patients}

This retrospective study was performed at our academic level 1 trauma center from January 2017 to December 2019. And it was approved by the institutional review committee of the ethics committee. Informed consent was provided by all the participants. All enrolled injuries were diagnosed as Schatzker type IV tibial plateau fractures with or without ACL avulsions. The exclusion criteria were: lacking of adequate radiographic or CT images, ipsilateral periarticular fracture (i.e., distal femoral fracture, femoral condylar fracture, or patellar fracture), open or pathological knee fractures, fractures in skeletally immature patients, or a history of knee surgery. After excluding unqualified patients, 99 patients were included. The study consisted of 56 men and 43 women, with an average age of 43.8 years (range, 25-70 years). The causes of injuries were traffic injuries in 56 cases, high falling injuries in 28 cases, and simple falling in 15 cases. The included patients performed a CT examination of the injured knee with a slice thickness of $0.6 \mathrm{~mm}$ (pixel spacing: $0.44 \times 0.44 \mathrm{~mm}$ ).

\section{Diagnosis of ACL Avulsions}

A senior orthopedist and a musculoskeletal radiologist were selected to confirm the diagnosis of ACL avulsions based on the image data. According to the displacement of ACL tibial attachment, they further subdivided ACL avulsions fractures into displaced ACL avulsions and nondisplaced ACL avulsions.

\section{D Fracture Models}

3D knee joint models of the subjects were generated by the Mimics software (version 20.0, Materialise, Leuven, Belgium). First, we imported the CT data (DICOM files) to the Mimics software. Second, the operation of segmentation was performed based on the preset bone threshold (unit $\geq$ 226). Tibial and femoral 3D models were constructed after smoothening and removing the patella.

\section{Standard Location of the 3D Model}

After measuring the midpoints of the medial-to-lateral and anterior-to-posterior widths of the tibia and femur, we could define the tibial axis line (TAL) and femoral axis line (FAL) in the coronal and sagittal planes. Femoral condyle line refers to the line between medial femoral condyle and lateral condyle in coronal plane. The axis of the posterior femoral condyle (PFCA) was determined by measuring the straight line of the posterior femoral condyle in the axial plane. Posterior tibial condylar axis (PTCA) is a tangent to the posterior edge of the tibial plateau. To satisfy the standard location of the 3D model, the femoral condylar line should be perpendicular to the TAL in the coronal plane, and the FAL should be parallel to the TAL in the sagittal plane by adjusting the position of the femur and tibia in the Mimics program. Meanwhile, the PFCA should be parallel to the PTCA in the axial plane to satisfy the standard location. All subjects adopted the standardized programs (-Fig. $\mathbf{1}$ ).
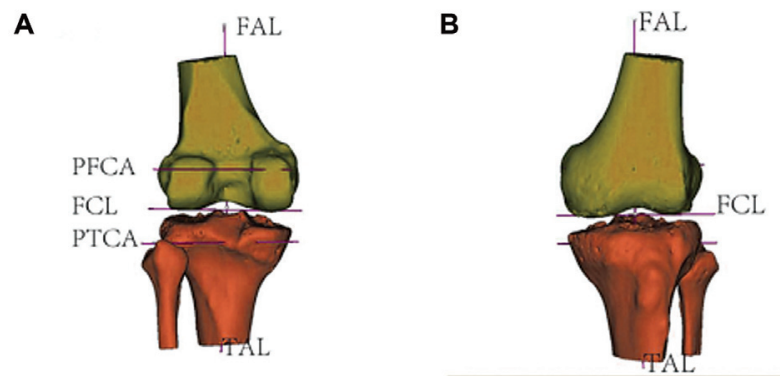

Fig. 1 The standard location of three-dimensional (3D) knee model. (A) Posterior view. (B) Anterior view. 
Simulating the Knee Position at the Time of Fracture The impingement of the femoral condyle can cause compression on the articular surface of the tibial plateau or tibial wedge fractures at the moment of fracture occurrence. We could simulate the knee posture by matching the compression degree of articular surface of tibial plateau and the morphology of femoral condyle. The procedure was completed by utilizing the relocation function in the Mimics software. The 3D tibial model was moved to match the tibial articular compression with the shape of femoral condyle, while the 3D femoral model remained stationary. The outlines of the tibia and femur were not allowed to intersect. Two attending physicians performed the procedures and a senior physician would make an exact conclusion when their opinions were divided on the location of the tibia (-Fig. 2).

\section{Quantitative 3D Measurements}

The 3-Matic Medical software (Materialise 12.0, Leuven, Belgium) was used for 3D measurements. After inputting the 3D femoral and tibial models of the standard location into the workspace of the 3-Matic software, the contours of the femur and tibia and the standard lines (TAL PFCA PTCA) were also projected into three planes of the 3-Matic software. Then the varus angles, valgus angles, flexion angles, and the tibial rotation angles could be measured by the software tools. The detailed angle measurements are as follows.

\section{Hyperextension, Extension, and Flexion Angles}

The measurements were performed in the sagittal plane. Both the location at the moment of fracture occurrence and the standard location were projected in the sagittal plane. The angles measured between the TALs in two different states were the basis of whether the knee was in hypertension, extension, or flexion. Knee hyperextension refers to a hyperextension angle of more than 5 degrees. Knee flexion refers to a flexion angle of more than 15 degrees. Knee extension refers to an angle between hyperextension and flexion (-Fig. $\mathbf{3}$ ).

\section{Valgus and Varus Angles}

The measurements were performed in the coronal plane. Both the location at the moment of fracture occurrence and the standard location were projected in the coronal plane. The angles measured between the TALs in two different states determined whether the knee is in a position of varus or valgus (-Figs. 4 and 5).

\section{External Rotation and Internal Rotation Angle}

The measurements were performed in the axial plane which demanded the projection of the PFCA and PTCA. The angles
A

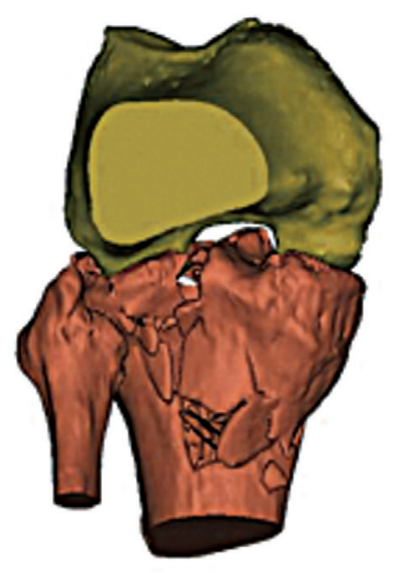

B

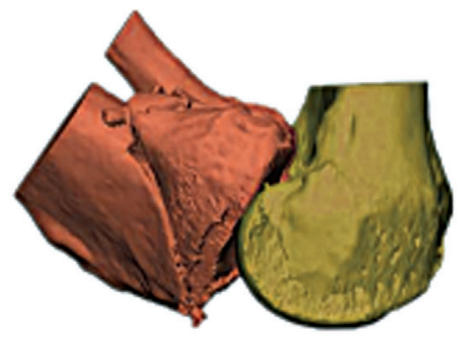


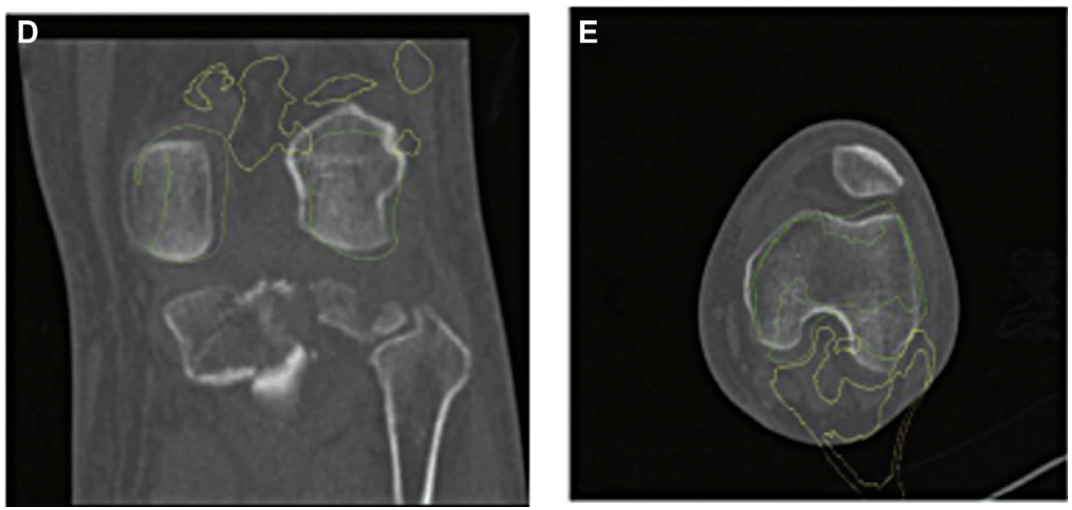

Fig. 2 The simulation of the knee posture at the moment of the fracture. (A) Sagittal plane in Mimics software. (B) Axial plane in Mimics software. (C) Axial plane in computed tomography (CT) image. (D) Sagittal plane in CT image. (E) Coronal plane in CT image. 


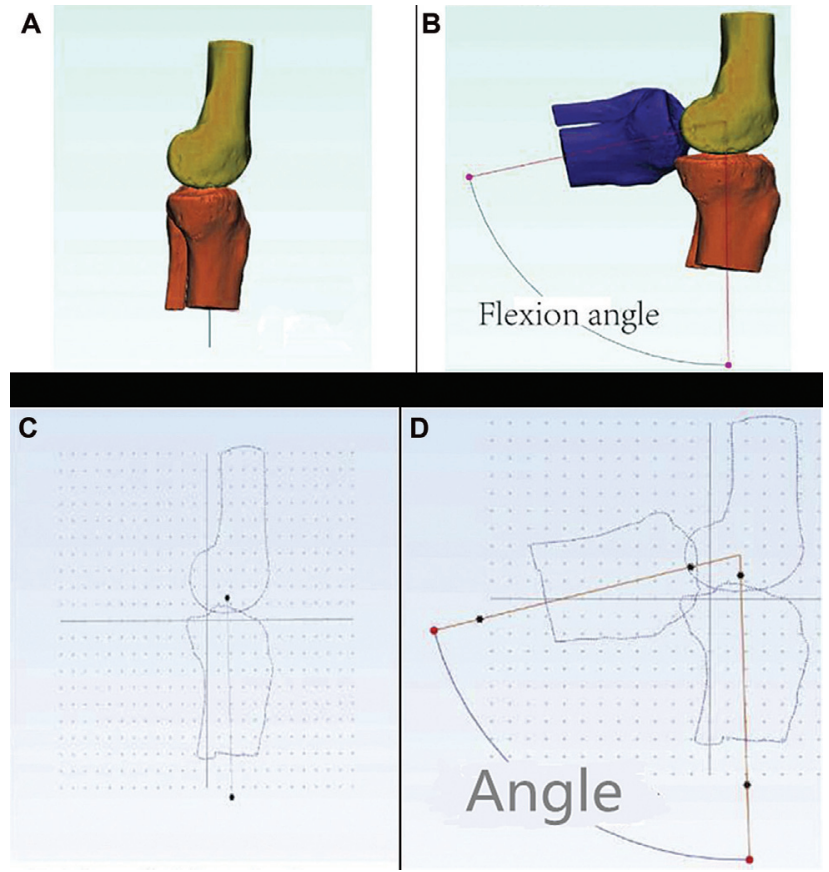

Fig. 3 The measurement of the flexion angle. (A) Standard lateral position in Mimics software. (B) Relocated tibial model (blue) and standard tibial model (brown) in Mimics software. (C) Standard lateral position in 3-Matic Medical software. (D) Measuring the angle to define "flexion" in 3-Matic Medical software. measured between the PFCA and PTCA determined whether the tibia is in internal or external rotation. An angle of rotation greater than 5 degrees refers to tibial internal or external rotation (-Fig. $\mathbf{6}$ ).

\section{Data Analysis}

All statistical data in our study were analyzed using the SPSS software (version 25.0). Chi-square tests and Fisher's exact tests were used to evaluate the difference between the classified data. A $p$-value of $<0.05$ was considered to be statistically significant.

\section{Results}

The relevant data of the 99 subjects were estimated by quantitative 3D measurements. According to the results of the angle measurements in three planes, 99 cases of Schatzker type IV tibial plateau fractures were divided into the following eight types of injury: hyperextension-varusinternal rotation, hyperextension-valgus-external rotation, extension-valgus, extension-varus, flexion-varus-internal rotation, flexion-varus-external rotation, flexion-valgus-internal rotation, and flexion-valgus-external rotation. Details of ACL are summarized in - Table 1.

No ACL avulsions were found among the injury pattern of hyperextension in our study. There were more associated ACL avulsions in the state of knee flexion (45 of 60) than knee
A
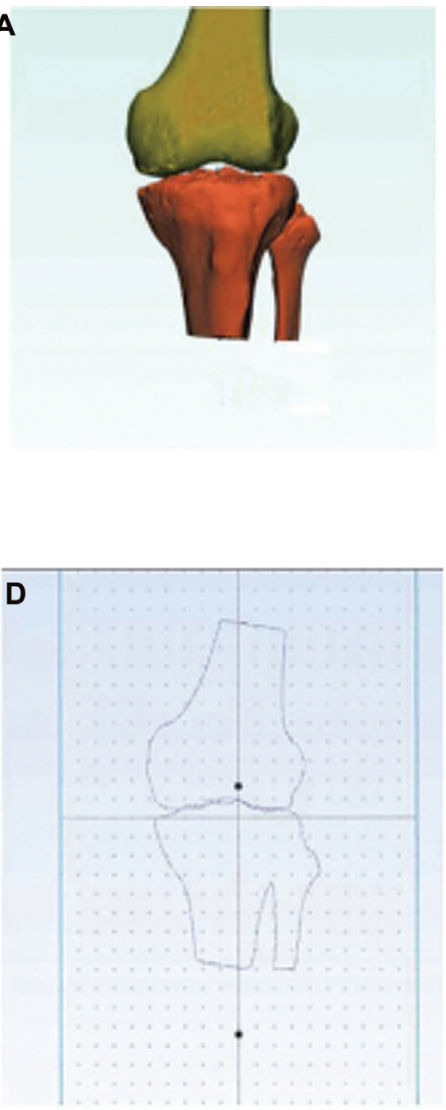

B



E

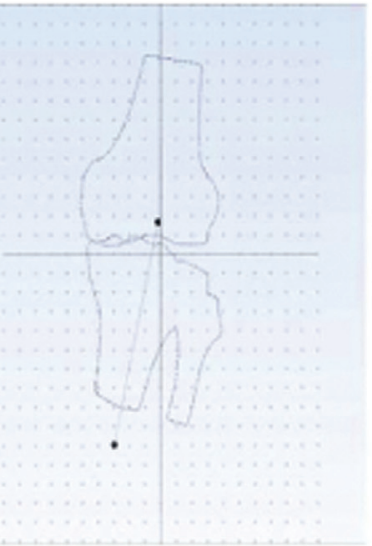

C



$\mathbf{F}$

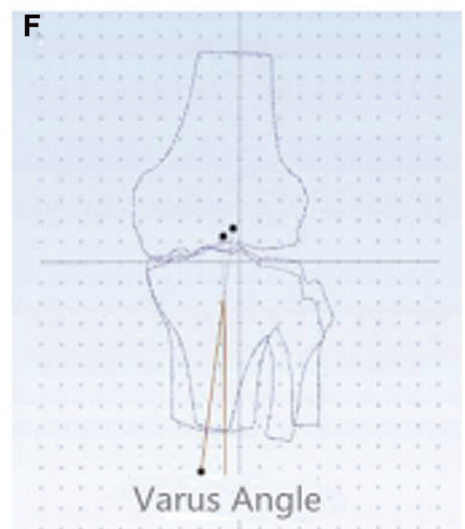

Fig. 4 The measurement of the varus angle. (A) Standard location in Mimics software. (B) Simulated injury pattern (varus) in Mimics software. (C) Combined mode in Mimics software. (D) Standard location in 3-Matic Medical software. (E) Simulated injury pattern (varus) in 3-Matic Medical software. (F) Combined mode in 3-Matic Medical software for measuring the angle to define "varus." 
A

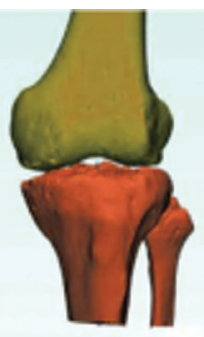

D

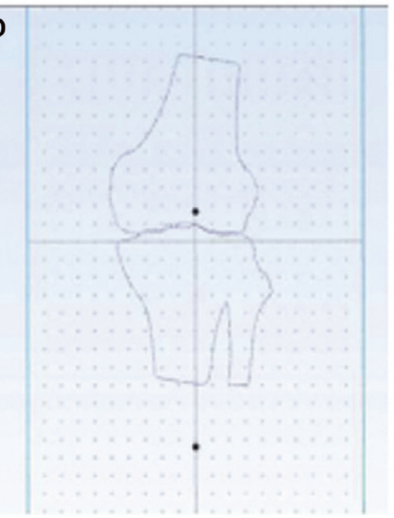

B
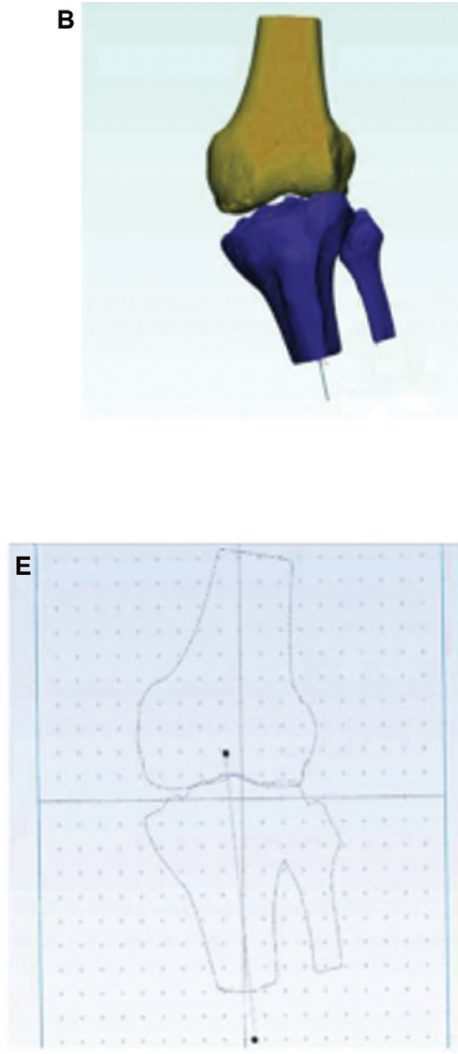

C

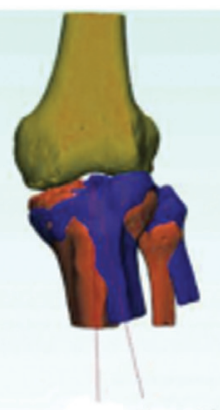

Fig. 5 The measurement of the valgus angle. (A) Standard location in Mimics software. (B) Simulated injury pattern (valgus) in Mimics software. (C) Combined mode in Mimics software. (D) Standard location in 3-Matic Medical software. (E) Simulated injury pattern (valgus) in 3-Matic Medical software. (F) Combined mode in 3-Matic Medical software for measuring the angle to define "valgus."

A

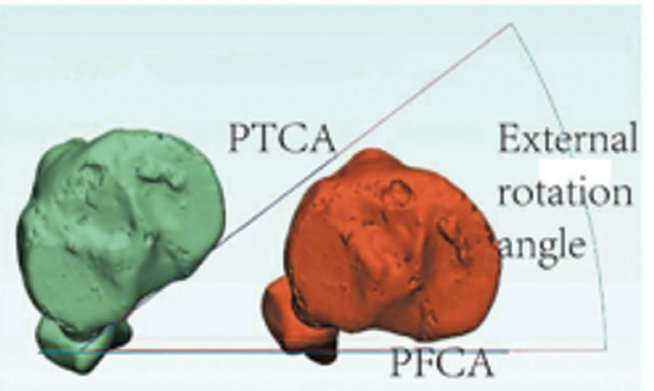

$x=$

C

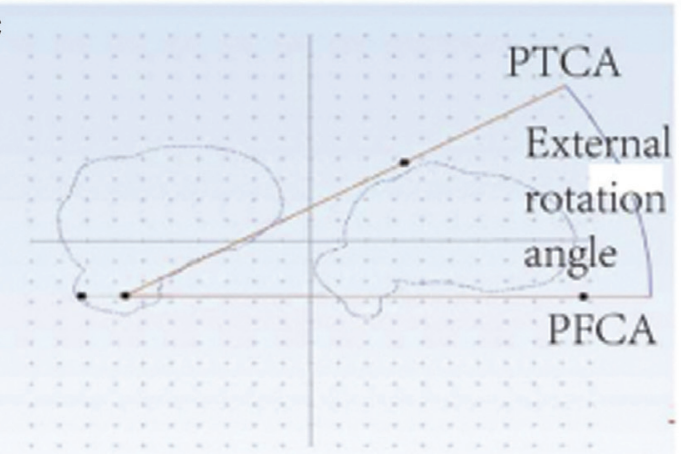

B

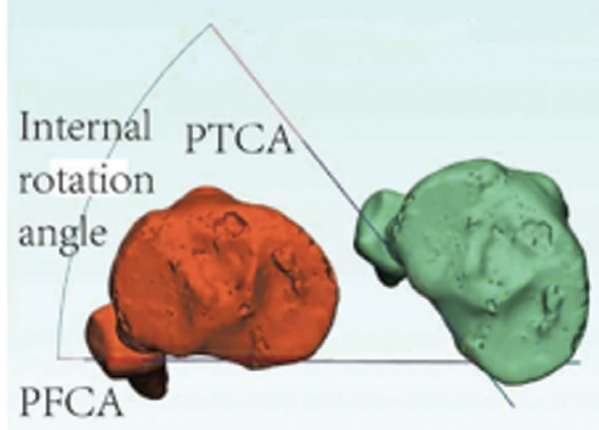

D

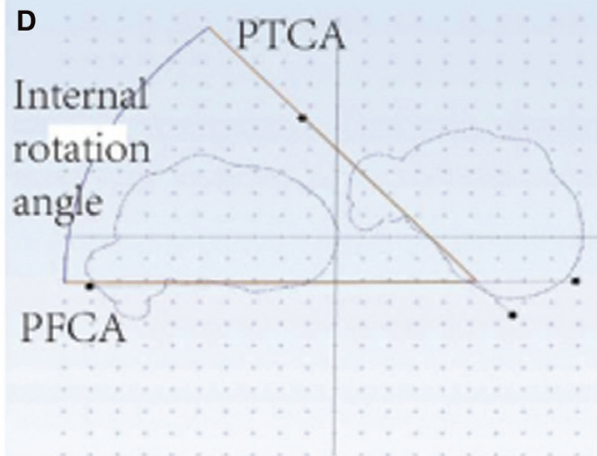

Fig. 6 The measurement of the internal and external angle. (A) External rotation of tibial in Mimics software. (B) Internal rotation of tibial in Mimics software. (C) The measurement of the external rotation angle in 3-Matic Medical software. (D) The measurement of the internal rotation angle in 3-Matic Medical software. 
Table 1 The cases of associated ACL avulsions among different injury patterns

\begin{tabular}{|l|l|l|l|l|l|}
\hline Position & Force & Rotation & Total case & ACL injury & Non-ACL injury \\
\hline \multirow{3}{*}{ Hyperextension } & Varus & Internal & 4 & 0 & 4 \\
\cline { 2 - 7 } & Valgus & External & 10 & 0 & 10 \\
\hline \multirow{2}{*}{ Extension } & Varus & None & 10 & 5 & 5 \\
\cline { 2 - 7 } & Valgus & None & 15 & 6 & 9 \\
\hline \multirow{3}{*}{ Flexion } & Varus & Internal & 11 & 7 & 4 \\
\cline { 2 - 7 } & & External & 12 & 7 & 5 \\
\cline { 2 - 6 } & \multirow{2}{*}{ Valgus } & Internal & 26 & 21 & 5 \\
\cline { 3 - 7 } & & External & 11 & 10 & 1 \\
\hline
\end{tabular}

Abbreviation: ACL, anterior cruciate ligament.

extension (11 of 25$)(p<0.05)$ in the setting of Schatzker type IV tibial plateau fracture. More associated ACL avulsions were found in the flexion-valgus injury patterns than the other injury patterns of same level which were called extension-valgus, extension-varus, and flexion-varus $(p<0.05)$. In terms of whether Schatzker type IV tibial plateau fracture was associated with ACL avulsion, there was no notable difference between the tibial internal and external rotation injury patterns $(p>0.05)$.

The classification of the injury patterns was summarized in - Table 2. There were more displaced ACL avulsion fractures which require surgical treatment in the state of knee flexion (32 of 45) than knee extension (3 of 11) $(p<0.05)$. Among patients with type III or IV ACL injuries, there was no significant difference between valgus force and varus force $(p>0.05)$. The tibial internal and external rotation showed no significant difference in producing displaced ACL avulsion fractures accompanied by Schatzker type IV tibial plateau fractures $(p>0.05)$.

\section{Discussion}

Schatzker type IV fractures are considered as high-energy injuries and associated soft tissue injuries are frequent. ${ }^{4,5,14}$ To gain further insight on this type of injury, Schatzker type IV fracture has been subtyped by many scholars. Wahlquist et al ${ }^{14}$ found that the more lateral the fracture line moves, the greater the risk of these associated soft tissue complications.
According to Chan et al's research, there was a high incidence of ACL injuries in the posteromedial tibial plateau fractures. ${ }^{15}$ Luo et al proposed a typical "two-column fracture" for traditional Schatzker type IV fractures (medial condylar fractures) ${ }^{16}$ Moore classified Schatzker type IV tibial plateau fractures into Moore type I or Moore type II. ${ }^{17}$ Based on these studies, ACL avulsion is one of the most common complications and should be taken seriously, especially the displaced fractures which require surgical repair. ${ }^{6,18}$ With a better understanding of the mechanism of injury, Schatzker type IV tibial plateau fractures can be diagnosed more comprehensively and appropriate treatment strategies can be selected by the orthopedists.

Although several articles had discussed the injury mechanism of various tibial plateau fractures, ${ }^{11-13,19-22}$ the injury mechanism of Schatzker type IV tibial plateau fracture with ACL avulsion has been rarely reported. We proposed a new experimental idea which studies how different knee positions may affect the prevalence of soft tissue injuries when the Schatzker IV fractures occur. In our study, we analyzed the injury mechanism of Schatzker type IV fractures with ACL avulsions by simulating the knee positions at the time of fractures, measuring the motion angles of tibial in the coronal and sagittal planes and selecting the cases of ACL avulsions. Based on the 3D models which were established by simulating the positions of tibia and femur at the time of fractures, the relevant data could be measured for investigating the injury mechanism.

Table 2 The distribution of associated ACL avulsions

\begin{tabular}{|l|l|l|l|l|l|}
\hline Position & Force & Rotation & Total case & \multicolumn{2}{|l|}{ Classification of ACL avulsions } \\
\cline { 4 - 6 } & & & & Nondisplaced & Displaced \\
\hline \multirow{3}{*}{ Extension } & Varus & None & 5 & 4 & 1 \\
\cline { 2 - 6 } & Valgus & None & 6 & 4 & 2 \\
\hline \multirow{3}{*}{ Flexion } & Varus & Internal & 7 & 3 & 4 \\
\cline { 3 - 6 } & & External & 7 & 2 & 5 \\
\cline { 2 - 6 } & Valgus & Internal & 21 & 5 & 16 \\
\cline { 3 - 6 } & & External & 10 & 3 & 8 \\
\hline
\end{tabular}

Abbreviation: ACL, anterior cruciate ligament. 
No ACL avulsions were found among the injury patterns of hyperextension in our study. Due to the morphology of the injury patterns which were characterized by anterior plateau articular depression involving the anterior rim, ACL avulsion may hardly happen among hyperextended knees when it is associated with Schatzker type IV fracture. More associated ACL avulsions were found in the state of knee flexion in our study, which has been supported by several researches. ${ }^{11-13,20}$ Meanwhile valgus force was the main cause of associated ACL avulsion in the study which would support the similar opinion of Wang et al. ${ }^{11}$ Patients with knees in the state of flexion at the moment of Schatzker type IV tibial plateau fracture should be taken seriously, especially under the impact of valgus force in clinical practices. Furthermore, more displaced ACL avulsion fractures which require surgical treatment were found in flexed knees (32 of 45) $(p<0.05)$. But, there was no significant difference in terms of force vectors, which means that the valgus force may produce more associated ACL avulsions, but it has little effect on the degree of injury in the setting of Schatzker type IV tibial plateau fractures.

In previous studies, the authors paid limited attention to tibial rotation when investigating the injury mechanism of tibial plateau fractures. ${ }^{11,13,20}$ In our study, tibial rotation was added as a factor to investigate the injury mechanism. According to the results of our study, tibial rotation was not a significant factor in both producing associated ACL avulsions and affecting the degree of injuries $(p>0.05)$.

There were some limitations in our study. Since the sample size of our study was relatively small, the results might be different if a larger sample size had been analyzed. Furthermore, due to the limited sample size, not all injury patterns may be included. In addition, translation of the tibia was not evaluated in this study, which needs for further studies.

\section{Conclusion}

In our study, a flexed knee at the moment of Schatzker type IV tibial plateau fracture occurrence is a high-risk factor for causing associated ACL avulsions and producing more displaced ACL avulsions. Meanwhile flexion-valgus pattern was the main cause of Schatzker type IV tibial plateau fractures associated with ACL avulsions. The findings of our study will help orthopedists to have a better understanding of the injury mechanism of Schatzker type IV tibial plateau fractures associated with ACL avulsions and then have predictive value in diagnosing and treating associated ACL avulsions to avoid unfavorable prognosis.

\section{Authors' Contributions}

Z.L. designed the research; S.W. and X.T. performed the research; A.P. wrote the paper. All authors read and approved the final version of the manuscript for publication.

\section{Ethical Approval}

This study was performed with the approval of the Clinical Ethical Committee of Third Hospital of Hebei Medical University. All procedures were strictly conducted in line with the code of ethics.

\section{Informed Consent}

All patients agreed and signed the informed consent.

Conflict of Interest

None declared.

\section{References}

1 Papagelopoulos PJ, Partsinevelos AA, Themistocleous GS, Mavrogenis AF, Korres DS, Soucacos PN. Complications after tibia plateau fracture surgery. Injury 2006;37(06):475-484

2 Kfuri M, Schatzker J. Revisiting the Schatzker classification of tibial plateau fractures. Injury 2018;49(12):2252-2263

3 Millar SC, Arnold JB, Thewlis D, Fraysse F, Solomon LB. A systematic literature review of tibial plateau fractures: what classifications are used and how reliable and useful are they? Injury 2018; 49(03):473-490

4 Schatzker J, McBroom R, Bruce D. The tibial plateau fracture. The Toronto experience 1968-1975. Clin Orthop Relat Res 1979; (138):94-104

5 Yan B, Sun J, Yin W. The prevalence of soft tissue injuries in operative Schatzker type IV tibial plateau fractures. Arch Orthop Trauma Surg 2020

6 Chouhan DK, Dhillon MS, John R, Khurana A. Management of neglected ACL avulsion fractures: a case series and systematic review. Injury 2017;48(2, Suppl 2):S54-S60

7 Meyers MH, McKeever FM. Fracture of the intercondylar eminence of the tibia. J Bone Joint Surg Am 1970;52(08): $1677-1684$

8 Blokker CP, Rorabeck CH, Bourne RB. Tibial plateau fractures. An analysis of the results of treatment in 60 patients. Clin Orthop Relat Res 1984;(182):193-199

9 Ali AM, Burton M, Hashmi M, Saleh M. Outcome of complex fractures of the tibial plateau treated with a beam-loading ring fixation system. J Bone Joint Surg $\mathrm{Br}$ 2003;85(05): 691-699

10 Stannard JP, Lopez R, Volgas D. Soft tissue injury of the knee after tibial plateau fractures. J Knee Surg 2010;23(04):187-192

11 Wang S, Peng AQ, Pan S, Hu YN, Zhang X, Gao JG. Analysis of medial tibial plateau fracture injury patterns using quantitative 3D measurements. J Orthop Sci 2020

12 Wang Y, Luo C, Zhu Y, et al. Updated Three-Column Concept in surgical treatment for tibial plateau fractures - a prospective cohort study of 287 patients. Injury 2016;47(07):1488-1496

13 Xie X, Zhan Y, Wang Y, Lucas JF, Zhang Y, Luo C. Comparative analysis of mechanism-associated 3-dimensional tibial plateau fracture patterns. J Bone Joint Surg Am 2020;102(05):410-418

14 Wahlquist M, Iaguilli N, Ebraheim N, Levine J. Medial tibial plateau fractures: a new classification system. J Trauma 2007;63(06): 1418-1421

15 Chan KK, Resnick D, Goodwin D, Seeger LL. Posteromedial tibial plateau injury including avulsion fracture of the semimembranous tendon insertion site: ancillary sign of anterior cruciate ligament tear at MR imaging. Radiology 1999;211(03):754-758

16 Luo CF, Sun H, Zhang B, Zeng BF. Three-column fixation for complex tibial plateau fractures. J Orthop Trauma 2010;24(11): 683-692

17 Moore TM. Fracture-dislocation of the knee. Clin Orthop Relat Res $1981 ;(156): 128-140$ 
18 Kuang SD, Su C, Zhao X, Li YS, Xiong YL, Gao SG. "Figure-of-Eight” suture-button technique for fixation of displaced anterior cruciate ligament avulsion fracture. Orthop Surg 2020;12(03):802-808

19 Bennett WF, Browner B. Tibial plateau fractures: a study of associated soft tissue injuries. J Orthop Trauma 1994;8(03): 183-188

20 Zhang BB, Sun $\mathrm{H}$, Zhan Y, et al. Reliability and repeatability of tibial plateau fracture assessment with an injury mechanism-based concept. Bone Joint Res 2019;8(08):357-366
21 Yang G, Zhu Y, Luo C, Putnis S. Morphological characteristics of Schatzker type IV tibial plateau fractures: a computer tomography based study. Int Orthop 2012;36(11):2355-2360

22 Zhai Q, Hu C, Xu Y, Wang D, Luo C. Morphologic study of posterior articular depression in Schatzker IV fractures. Orthopedics 2015; 38(02):e124-e128 